\title{
Coupled valence bond theory
}

\author{
Remco W.A. Havenith * \\ Debye Institute, Theoretical Chemistry Group, Utrecht University, Padualaan 8, 3584 CH Utrecht, The Netherlands
}

Received 11 July 2005; in final form 8 August 2005

Available online 30 August 2005

\begin{abstract}
In this Letter, the formulation and implementation of a parallel response property code for non-orthogonal, valence bond wavefunctions are described. Test calculations on benzene and cyclobutadiene show that the polarisability and magnetisability tensors obtained using valence bond theory are comparable to those obtained at the full $\pi$-CASSCF level of theory. The properties are a weighted average of those of its individual resonance structures. Resonance has no relevance for the polarisability and magnetisability. (C) 2005 Elsevier B.V. All rights reserved.
\end{abstract}

\section{Introduction}

The evaluation of molecular properties has become a standard tool in computational chemistry. For all kinds of wavefunctions, ranging from Hartree-Fock to CI and $\operatorname{CCSD}(\mathrm{T})$, property codes have been implemented. For valence bond wavefunctions, however, only for the special case of a spin-coupled wavefunction, the theory and implementation has been realised [1]. In valence bond theory, a bond is formed by spin-coupling two electrons, each occupying an non-orthogonal, atomic orbital. This allows an extreme flexibility in the formulation of wavefunctions and orbital models. The wavefunction may be written as a linear superposition of structures which bear a close resemblance to Lewis-structures; bonding can directly be analysed in terms of resonance between covalent and ionic descriptions. The availability of a second-order VB response code would offer the possibility to study the effects of orbital delocalisation and of resonance between multiple structures on response properties, like the polarisability, and magnetisability. This may serve for judging the quality of the (restricted) wavefunction.

The implementation of the ab initio VB method in TURTLE $[2,3]$ is more general then the spin-coupled ap-

\footnotetext{
${ }^{*}$ Fax: +31302537504.

E-mail address: r.w.a.havenith@chem.uu.nl.
}

proach. For example, not all possible spin-coupling schemes have to be considered, and different orbitals for different structures can be chosen [4]. In this Letter, the formulation and implementation in TURTLE, of a non-orthogonal, static, response property code is discussed using the derivative technique. As will be seen, the matrix elements required to evaluate the electronic Hessian, and property gradient, are identical to those needed for the evaluation of frequency-dependent properties, using propagator techniques [5]. Thus, the results obtained here can be used in the development of VB frequency-dependent properties. To show the applicability of this approach, the polarisabilities and magnetisabilities of benzene, cyclobutadiene and their localised, one-structure descriptions have been calculated.

\section{Theory and implementation}

In valence bond theory, the wavefunction $\Psi_{0}$ is written as a linear combination of structures

$\Psi_{0}=\sum_{n} C_{n} \Phi_{n}$.

Each structure is written as a fixed combination of determinants $\Phi_{n}=\sum_{i} c_{i} \Delta_{i}$ representing, for example, the desired spin-coupling scheme. The orbitals $(\phi)$ used to build the determinants are not necessarily orthogonal, 
and may be either fixed or optimised with or without constraints. The structure coefficients $C_{n}$ are variationally determined in a CI-like fashion. The orbitals are optimised by mixing them with virtual orbitals until the generalised Brillouin condition [6] is satisfied. Both the virtual orbitals and orbital mixings are determined by the imposed restrictions on the wavefunction $[7,8]$. A valence bond wavefunction is thus optimised with respect to the CI coefficients $C_{n}$ and orbital rotations $\kappa$ (see $[3,7,8]$ ). For the evaluation of second-order response properties, at least the derivatives of the wavefunction with respect to those orbital rotations used in the orbital optimisation, and with respect to the CI coefficients are required.

Following coupled Hartree-Fock theory [5,9], the Hamiltonian in the presence of a basis set independent perturbation, like the electric or magnetic field, becomes $\hat{H}=\hat{H}_{0}+\lambda \hat{V}_{1}+\lambda^{2} \hat{V}_{2}$, in which $\lambda$ is the perturbation strength. The energy, as a function of $\lambda$, is then $E(\lambda)=\left\langle\Psi(\lambda)\left|\hat{H}_{0}+\lambda \hat{V}_{1}+\lambda^{2} \hat{V}_{2}\right| \Psi(\lambda)\right\rangle /\langle\Psi(\lambda) \mid \Psi(\lambda)\rangle$.

In the following derivation of the VB response property theory, no assumptions are made about normalisation and orthogonality of orbitals. The first and second derivatives of the energy with respect to the perturbation parameter $\lambda$, using the quotient rule, become

$\left.\frac{\partial E}{\partial \lambda}\right|_{\lambda=0}=2 \frac{\left\langle\frac{\partial \Psi_{0}}{\partial \lambda}\left|\hat{H}-E_{0}\right| \Psi_{0}\right\rangle}{\left\langle\Psi_{0} \mid \Psi_{0}\right\rangle}+\frac{\left\langle\Psi_{0}\left|\hat{V}_{1}\right| \Psi_{0}\right\rangle}{\left\langle\Psi_{0} \mid \Psi_{0}\right\rangle}$

and

$$
\begin{aligned}
\left.\frac{\partial^{2} E}{\partial \lambda^{2}}\right|_{\lambda=0}= & 2 \frac{\left\langle\frac{\partial^{2} \Psi_{0}}{\partial \lambda^{2}}\left|\hat{H}-E_{0}\right| \Psi_{0}\right\rangle}{\left\langle\Psi_{0} \mid \Psi_{0}\right\rangle}+2 \frac{\left\langle\frac{\partial \Psi_{0}}{\partial \lambda}\left|\hat{H}-E_{0}\right| \frac{\partial \Psi_{0}}{\partial \lambda}\right\rangle}{\left\langle\Psi_{0} \mid \Psi_{0}\right\rangle} \\
& +4 \frac{\left\langle\frac{\partial \Psi_{0}}{\partial \lambda}\left|\hat{V}_{1}-V_{1}^{0}\right| \Psi_{0}\right\rangle}{\left\langle\Psi_{0} \mid \Psi_{0}\right\rangle} \\
& -8 \frac{\left\langle\frac{\partial \Psi_{0}}{\partial \lambda}\left|\hat{H}-E_{0}\right| \Psi_{0}\right\rangle\left\langle\frac{\partial \Psi_{0}}{\partial \lambda} \mid \Psi_{0}\right\rangle}{\left\langle\Psi_{0} \mid \Psi_{0}\right\rangle^{2}}+2 \frac{\left\langle\Psi_{0}\left|\hat{V}_{2}\right| \Psi_{0}\right\rangle}{\left\langle\Psi_{0} \mid \Psi_{0}\right\rangle} .
\end{aligned}
$$

In the last equation, use is made of the definition $V_{1}^{0}=\left\langle\Psi_{0}\left|\hat{V}_{1}\right| \Psi_{0}\right\rangle /\left\langle\Psi_{0} \mid \Psi_{0}\right\rangle$, analogous to $E_{0}$. The wavefunction parameters $C$ (orbital coefficients and VB structure coefficients) depend on the perturbation, and thus this equation can be rewritten using $\partial / \partial \lambda=(\partial C /$ $\partial \lambda)(\partial / \partial C)$ as

$$
\begin{aligned}
\left.\frac{\partial^{2} E}{\partial \lambda^{2}}\right|_{\lambda=0}= & \left(\frac{\partial C}{\partial \lambda}\right)\left[2 \frac{\left\langle\frac{\partial^{2} \Psi_{0}}{\partial \lambda \partial C}\left|\hat{H}-E_{0}\right| \Psi_{0}\right\rangle}{\left\langle\Psi_{0} \mid \Psi_{0}\right\rangle}\right. \\
& +2 \frac{\left\langle\frac{\partial \Psi_{0}}{\partial C}\left|\hat{H}-E_{0}\right| \frac{\partial \Psi_{0}}{\partial \lambda}\right\rangle}{\left\langle\Psi_{0} \mid \Psi_{0}\right\rangle}+4 \frac{\left\langle\frac{\partial \Psi_{0}}{\partial C}\left|\hat{V}_{1}-V_{1}^{0}\right| \Psi_{0}\right\rangle}{\left\langle\Psi_{0} \mid \Psi_{0}\right\rangle} \\
& -4 \frac{\left\langle\frac{\partial \Psi_{0}}{\partial C}\left|\hat{H}-E_{0}\right| \Psi_{0}\right\rangle\left\langle\frac{\partial \Psi_{0}}{\partial \lambda} \mid \Psi_{0}\right\rangle}{\left\langle\Psi_{0} \mid \Psi_{0}\right\rangle^{2}} \\
& \left.-4 \frac{\left\langle\frac{\partial \Psi_{0}}{\partial \lambda}\left|\hat{H}-E_{0}\right| \Psi_{0}\right\rangle\left\langle\frac{\partial \Psi_{0}}{\partial C} \mid \Psi_{0}\right\rangle}{\left\langle\Psi_{0} \mid \Psi_{0}\right\rangle^{2}}\right]+2 \frac{\left\langle\Psi_{0}\left|\hat{V}_{2}\right| \Psi_{0}\right\rangle}{\left\langle\Psi_{0} \mid \Psi_{0}\right\rangle} .
\end{aligned}
$$

In analogy with CHF theory, the wavefunction should be stationary when the perturbation is applied, thus the derivative of the stationary state condition with respect to the perturbation parameter should equal zero. This condition leads to the coupled VB equations:

$$
\begin{aligned}
\frac{\partial}{\partial \lambda}( & \left.\frac{\partial}{\partial C} \frac{\left\langle\Psi_{0}\left|\hat{H}_{0}+\lambda \hat{V}_{1}+\lambda^{2} \hat{V}_{2}\right| \Psi_{0}\right\rangle}{\left\langle\Psi_{0} \mid \Psi_{0}\right\rangle}\right)\left.\right|_{\lambda=0} \\
= & 2 \frac{\left\langle\frac{\partial \Psi_{0}}{\partial C \partial \lambda}\left|\hat{H}-E_{0}\right| \Psi_{0}\right\rangle}{\left\langle\Psi_{0} \mid \Psi_{0}\right\rangle}+2 \frac{\left\langle\frac{\partial \Psi_{0}}{\partial C}\left|\hat{H}-E_{0}\right| \frac{\partial \Psi_{0}}{\partial \lambda}\right\rangle}{\left\langle\Psi_{0} \mid \Psi_{0}\right\rangle} \\
& +2 \frac{\left\langle\frac{\partial \Psi_{0}}{\partial C}\left|\hat{V}_{1}-V_{1}^{0}\right| \Psi_{0}\right\rangle}{\left\langle\Psi_{0} \mid \Psi_{0}\right\rangle}-4 \frac{\left\langle\frac{\partial \Psi_{0}}{\partial C}\left|\hat{H}-E_{0}\right| \Psi_{0}\right\rangle\left\langle\frac{\partial \Psi_{0}}{\partial \lambda} \mid \Psi_{0}\right\rangle}{\left\langle\Psi_{0} \mid \Psi_{0}\right\rangle^{2}} \\
& -4 \frac{\left\langle\frac{\partial \Psi_{0}}{\partial \lambda}\left|\hat{H}-E_{0}\right| \Psi_{0}\right\rangle\left\langle\frac{\partial \Psi_{0}}{\partial C} \mid \Psi_{0}\right\rangle}{\left\langle\Psi_{0} \mid \Psi_{0}\right\rangle^{2}}=0 .
\end{aligned}
$$

Eq. (5) can be simplified to the working response equation by using the fact that the wavefunction is optimised and normalised.

$$
\begin{aligned}
& \left(\frac{\partial C}{\partial \lambda}\right)\left[\left\langle\frac{\partial^{2} \Psi_{0}}{\partial C^{2}}\left|\hat{H}-E_{0}\right| \Psi_{0}\right\rangle+\left\langle\frac{\partial \Psi_{0}}{\partial C}\left|\hat{H}-E_{0}\right| \frac{\partial \Psi_{0}}{\partial C}\right\rangle\right] \\
& \quad+\left\langle\frac{\partial \Psi_{0}}{\partial C}\left|\hat{V}_{1}-V_{1}^{0}\right| \Psi_{0}\right\rangle=0 .
\end{aligned}
$$

From this equation, $\partial C / \partial \lambda$ can be determined. The response equation can be written in matrix notation as $\mathbf{u} \mathbf{G}=-\mathbf{F}$, where $\mathbf{u}(\partial C / \partial \lambda)$ is the change of the wavefunction due to the perturbation, $\mathbf{G}\left(\left\langle\frac{\partial^{2} \Psi_{0}}{\partial C^{2}}\right| \hat{H}-\right.$ $\left.E_{0}\left|\Psi_{0}\right\rangle+\left\langle\frac{\partial \Psi_{0}}{\partial C}\left|\hat{H}-E_{0}\right| \frac{\partial \Psi_{0}}{\partial C}\right\rangle\right)$ is the second derivative (electronic Hessian) of the energy with respect to the wavefunction parameters, and $\mathbf{F}\left(\left\langle\frac{\partial \Psi_{0}}{\partial C}\left|\hat{V}_{1}-V_{1}^{0}\right| \Psi_{0}\right\rangle\right)$ is the property gradient.

The combination of Eqs. (4) and (5) by identification of identical terms, and taking normalisation into account, leads to a simplified formula for the second-order property $(P)$

$P=\left.\frac{\partial^{2} E}{\partial \lambda^{2}}\right|_{\lambda=0}=2\left(\frac{\partial C}{\partial \lambda}\right)\left\langle\frac{\partial \Psi_{0}}{\partial C}\left|\hat{V}_{1}-V_{1}^{0}\right| \Psi_{0}\right\rangle+2\left\langle\Psi_{0}\left|\hat{V}_{2}\right| \Psi_{0}\right\rangle$,

which can be written in matrix notation as $P=2 \mathbf{u F}+2 V_{2}^{0}$.

Using the derivative of the wavefunction with respect to the orbital rotations $\kappa_{i a}$, which is $\partial \Psi / \partial \kappa_{i a}=E_{i a} \Psi$, where $E_{i a}$ is the excitation operator that replaces orbital $a$ for $i$, once for $\alpha$ spin and once for $\beta$ spin, the orbital part of the property gradient (F) can be written explicitly in matrix elements as

$$
\left(\frac{\partial}{\partial \kappa_{i a}}\right)\left(\frac{\left\langle\Psi_{0}\left|\hat{V}_{1}\right| \Psi_{0}\right\rangle}{\left\langle\Psi_{0} \mid \Psi_{0}\right\rangle}\right)=2\left\langle E_{i a} \Psi_{0}\left|\hat{V}_{1}-V_{1}^{0}\right| \Psi_{0}\right\rangle=F_{i a}
$$

and the CI part (see Eq. (1)) as

$$
\left(\frac{\partial}{\partial C_{n}}\right)\left(\frac{\left\langle\Psi_{0}\left|\hat{V}_{1}\right| \Psi_{0}\right\rangle}{\left\langle\Psi_{0} \mid \Psi_{0}\right\rangle}\right)=2\left\langle\Phi_{n}\left|\hat{V}_{1}-V_{1}^{0}\right| \Psi_{0}\right\rangle=F_{n}
$$


The property gradient is evaluated for all possible orbital rotations $\kappa_{i a}$, and if $F_{i a}=0$ for a particular orbital rotation, then all derivatives involving this rotation are neglected in the evaluation of the Hessian. The rotations for which $F_{i a} \neq 0$, are tested against the imposed restrictions on the wavefunction: if strictly atomic orbitals are used, and this rotation corresponds to an excitation from one centre to another centre, the derivatives involving this rotation are again omitted from the Hessian.

In the special case that symmetry leads to a $\sigma$ - and $\pi$ system, e.g., benzene, where the $\pi$-system can easily be described by strictly atomic orbitals, whereas the $\sigma$-system is composed of delocalised, Hartree-Fock like orbitals, orbital rotations that mix the $\sigma$ and $\pi$ orbitals are not ignored, as the $\sigma$ orbitals do not obey any imposed restriction.

The electronic Hessian (G) can be obtained by differentiating the energy expression $E=\left\langle\Psi_{0}\left|\hat{H}_{0}\right| \Psi_{0}\right\rangle /\left\langle\Psi_{0} \mid \Psi_{0}\right\rangle$ twice with respect to the wavefunction parameters (analogous to the derivation of Eq. (5))

$$
\begin{aligned}
\frac{\partial^{2} E}{\partial X_{1} \partial X_{2}}= & 2\left\langle\frac{\partial^{2} \Psi_{0}}{\partial X_{1} \partial X_{2}}\left|\hat{H}-E_{0}\right| \Psi_{0}\right\rangle \\
& +2\left\langle\frac{\partial \Psi_{0}}{\partial X_{1}}\left|\hat{H}-E_{0}\right| \frac{\partial \Psi_{0}}{\partial X_{2}}\right\rangle \\
& -4\left\langle\frac{\partial \Psi_{0}}{\partial X_{1}}\left|\hat{H}-E_{0}\right| \Psi_{0}\right\rangle\left\langle\frac{\partial \Psi_{0}}{\partial X_{2}} \mid \Psi_{0}\right\rangle \\
& -4\left\langle\frac{\partial \Psi_{0}}{\partial X_{2}}\left|\hat{H}-E_{0}\right| \Psi_{0}\right\rangle\left\langle\frac{\partial \Psi_{0}}{\partial X_{1}} \mid \Psi_{0}\right\rangle
\end{aligned}
$$

in which $X_{n}$ denotes differentiation with respect to either orbital rotations or CI coefficients. Note that the last two terms of Eq. (10) vanish because of the fact that the wavefunction is optimised. The orbital-orbital block $\left(\mathbf{G}_{i a, j b}\right)$ of the electronic Hessian therefore can be written as

$$
\begin{aligned}
\frac{\partial^{2} E}{\partial \kappa_{i a} \partial \kappa_{j b}}= & 2\left\langle E_{i a} E_{j b} \Psi_{0}\left|\hat{H}-E_{0}\right| \Psi_{0}\right\rangle \\
& +2\left\langle E_{i a} \Psi_{0}\left|\hat{H}-E_{0}\right| E_{j b} \Psi_{0}\right\rangle,
\end{aligned}
$$

while the CI-CI block ( $\mathbf{G}_{n, m}$, see Eq. (1)) becomes [10]

$$
\frac{\partial^{2} E}{\partial C_{n} \partial C_{m}}=2\left\langle\Phi_{n}\left|\hat{H}-E_{0}\right| \Phi_{m}\right\rangle \text {. }
$$

Lastly, the mixed orbital-CI block $\left(\mathbf{G}_{i a, n}\right)$ equals

$$
\frac{\partial^{2} E}{\partial C_{n} \partial \kappa_{i a}}=2\left\langle E_{i a} \Phi_{n}\left|\hat{H}-E_{0}\right| \Psi_{0}\right\rangle+2\left\langle\Phi_{n}\left|\hat{H}-E_{0}\right| E_{i a} \Psi_{0}\right\rangle .
$$

Note that if all orbitals are mutually orthogonal, the terms containing $E_{0}$ vanish, and that these equations reduce to the familiar orthogonal expressions [11].

For the calculation of the electric field response, $\hat{V}_{1}$ contains the dipole operator $(\hat{x}, \hat{y}$, and $\hat{z})$, and $\hat{V}_{2}=0$. In the case of magnetic field perturbations, $\hat{V}_{1}$ is complex, resulting in antisymmetric property matrices; the formulas for the electronic Hessian are given in Appendix A.

All required matrix elements for the $\mathbf{F}$ and $\mathbf{G}$, and $\left\langle\Psi_{0}\left|\hat{V}_{2}\right| \Psi_{0}\right\rangle$ matrices are evaluated according to the generalised Slater-Condon rules [12,13], which are already implemented in TURTLE (also in a parallel version [14]). These matrix elements in structure basis can be expressed in determinant basis, and parallelism is achieved by dividing the calculation of the matrix elements on determinant basis amongst the processors [14]. The linear set of equations Eq. (5) is solved for $\mathbf{u}$, and finally, the desired property is calculated according to Eq. (7).

\section{Sample calculations}

Geometries of benzene $\left(\mathbf{1}(\mathbf{A}+\mathbf{B}), \mathrm{D}_{6 \mathrm{~h}}\right)$, and cyclobutadiene $\left(\mathbf{2}(\mathbf{A}+\mathbf{B}), \mathbf{D}_{2 \mathrm{~h}}\right)$ were optimised at the RHF/6-31G level of theory using GAMESS-UK [15]. This basis set was used in all further calculations. Two valence bond models for description of the $\pi$-system were employed: (1) the $\pi$-system was composed of strictly atomic p-orbitals ('strictly atomic model'), and (2) the $\pi$-system was composed of p-orbitals which are predominantly atomic in nature, but are allowed to have tails on neighbouring atoms ('delocal model') (cf. [16]). The starting $\sigma$-orbitals were taken from a preceding RHF calculation. All orbitals were optimised in all calculations.

For comparison, polarisabilities and magnetisabilities were calculated at the full $\pi$ CASSCF/6-31G level of theory, for benzene, and cyclobutadiene with DALTON [17].

At the RHF/6-31G optimised geometry, two different wavefunctions were employed for both molecules. One in which both Kekulé structures were considered, and one in which only one Kekulé structure was used. For both wavefunctions, both orbital models were employed.

The calculations are denoted by $\mathbf{n}(\mathbf{S})$, where $\mathbf{n}$ refers to the compound number and (S) refers to the structure (see Fig. 1). The results obtained for energies, resonance energies, and weights of the various structures of all

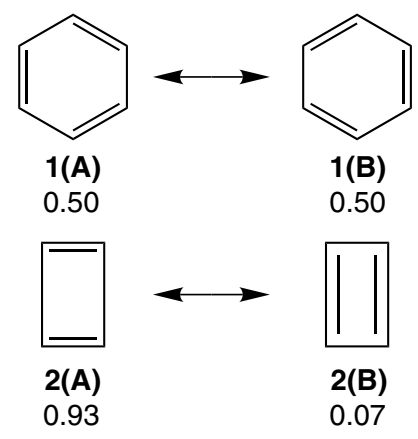

Fig. 1. The definition of the structures, and their weights in the VBSCF delocal wavefunction. 
calculations are in agreement with literature data $[16,18,19]$.

\section{Results and discussion}

Table 1 presents the polarisability $(\alpha)$ tensors calculated for 1,3,5-cyclohexatriene $(\mathbf{1}(\mathbf{A}))$, and benzene $(\mathbf{1}(\mathbf{A}+\mathbf{B}))$, and Table 2 those for cyclobutadiene $(\mathbf{2}(\mathbf{A}+\mathbf{B}))$. A comparison of the polarisability tensors shows that the delocal VB results are in close agreement with those obtained using the CASSCF or spin-coupled [20] wavefunctions. For the strictly atomic model, only the out-of-plane $\left(\alpha_{z z}\right)$ components are in line with the CASSCF results, whereas the in-plane $\left(\alpha_{x x}, \alpha_{y y}\right)$ components are considerably smaller for both $\mathbf{1}$ and $\mathbf{2}$.

The out-of-plane $\left(\alpha_{z z}\right)$ component is governed by $\left(\sigma \rightarrow \pi^{*}\right)$ - and $\left(\pi \rightarrow \sigma^{*}\right)$-type excitations, while the in-plane components by $\left(\sigma \rightarrow \sigma^{*}\right)$ - and $\left(\pi \rightarrow \pi^{*}\right)$-type

Table 1

The polarisability $(\alpha)$ tensors (a.u.) for 1,3,5-cyclohexatriene $(\mathbf{1}(\mathbf{A}))$, and benzene $(\mathbf{1}(\mathbf{A}+\mathbf{B}))$

\begin{tabular}{|c|c|c|c|c|c|c|}
\hline \multirow[t]{2}{*}{ Model } & \multicolumn{3}{|c|}{$\begin{array}{l}\text { 1,3,5-Cyclohexatriene } \\
\text { (1(A)) }\end{array}$} & \multicolumn{3}{|c|}{ Benzene $(\mathbf{1}(\mathbf{A}+\mathbf{B}))$} \\
\hline & $\alpha_{x x}, \alpha_{y y}$ & $\alpha_{z z}$ & $\alpha_{\mathrm{av}}$ & $\alpha_{x x}, \alpha_{y y}$ & $\alpha_{z z}$ & $\alpha_{\mathrm{av}}$ \\
\hline AS & - & - & - & 62.53 & 18.14 & 47.7 \\
\hline Strictly at & 41.48 & 19.44 & 34.13 & 41.40 & 19.29 & 34. \\
\hline Delocal & 62.91 & 18.20 & 48.01 & 61.15 & 18.14 & 46.81 \\
\hline
\end{tabular}

excitations. In the strictly atomic model, rotations of one $\mathrm{p}_{\pi}$-orbital to another $\mathrm{p}_{\pi}$-orbital located on a different atom are excluded. The out-of-plane component obtained in the strictly atomic model is thus not influenced by this restriction. The in-plane components, however, are affected; the contribution of the $\sigma$-orbitals is not influenced, but the $\pi$-contribution would vanish if the atomic p-orbitals on each centre were orthogonal. Because of the overlap between the orbitals, these type of excitations still contribute to the overall polarisability tensor.

1,3,5-Cyclohexatriene $(\mathbf{1}(\mathbf{A}))$, which has 'localised' bonds, has a similar polarisability to benzene $(\mathbf{1}(\mathbf{A}+\mathbf{B}))$, which has 'delocalised' bonds, due to resonance. The small differences between the polarisability tensor of 1,3,5-cyclohexatriene and that of benzene can only be attributed to the small differences in orbitals. Already a consideration of symmetry reveals that resonance does not play a major role in the properties. The CI response is proportional to $\left\langle\Psi_{0}|\hat{O}| \Psi_{1}\right\rangle$. The groundstate wavefunction $\left(\Psi_{0}=N\left(\Phi_{1}+\Phi_{2}\right)\right)$ possesses $\mathrm{A}_{1 \mathrm{~g}}$ symmetry, and the only excited state $\left(\Psi_{1}=N\left(\Phi_{1}-\Phi_{2}\right)\right)$ has $\mathrm{B}_{2 \mathrm{u}}$ symmetry. As the symmetries of the dipole moment operators are $\mathrm{E}_{1 \mathrm{u}}$ and $\mathrm{A}_{2 \mathrm{u}}$, the $\mathrm{CI}$ response vanishes by symmetry. The polarisabilities of benzene and cyclobutadiene are in this model thus the weighted averages of the polarisability of each individual resonance structure.

As for benzene, the cyclobutadiene response properties for one and two structures do not differ significantly.

Table 2

The polarisability $(\alpha)$ tensors (a.u.) for 1,3-cyclobutadiene (2(A)), and between parentheses of structure $\mathbf{2 B}$, and cyclobutadiene (2(A $+\mathbf{B}))$

\begin{tabular}{|c|c|c|c|c|c|c|}
\hline \multirow[t]{2}{*}{ Model } & \multicolumn{3}{|c|}{ 1,3-Cyclobutadiene $(\mathbf{2}(\mathbf{A}))(\mathbf{2}(\mathbf{B}))$} & \multicolumn{3}{|c|}{ Cyclobutadiene $(\mathbf{2}(\mathbf{A}+\mathbf{B}))$} \\
\hline & $0.5\left(\alpha_{x x}+\alpha_{y y}\right)$ & $\alpha_{z z}$ & $\alpha_{\mathrm{av}}$ & $0.5\left(\alpha_{x x}+\alpha_{y y}\right)$ & $\alpha_{z z}$ & $\alpha_{\mathrm{av}}$ \\
\hline $\operatorname{CASSCF}(4,4)$ & - & - & - & 37.95 & 13.64 & 29.84 \\
\hline Strictly atomic & $30.97(30.80)$ & $14.04(13.82)$ & $25.33(25.14)$ & 30.96 & 13.94 & 25.29 \\
\hline Delocal & $37.97(38.87)$ & $13.66(13.61)$ & $29.87(30.45)$ & 37.89 & 13.63 & 29.80 \\
\hline
\end{tabular}

Table 3

The magnetisability $(\chi)$ tensors (a.u.) for 1,3,5-cyclohexatriene $(\mathbf{1}(\mathbf{A}))$, benzene $(\mathbf{1}(\mathbf{A}+\mathbf{B}))$, 1,3-cyclobutadiene $(\mathbf{2}(\mathbf{A}))$, and between parentheses of structure 2B, and cyclobutadiene $(\mathbf{2}(\mathbf{A}+\mathbf{B}))$

\begin{tabular}{|c|c|c|c|c|c|c|}
\hline \multirow[t]{2}{*}{ Model } & \multicolumn{3}{|c|}{ 1,3,5-Cyclohexatriene $(\mathbf{1}(\mathbf{A}))$} & \multicolumn{3}{|c|}{ Benzene $(\mathbf{1}(\mathbf{A}+\mathbf{B}))$} \\
\hline & $\chi_{x x}, \chi_{y y}$ & $\chi_{z z}$ & $\chi_{\mathrm{av}}$ & $\chi_{x x}, \chi_{y y}$ & $\chi_{z z}$ & $\chi_{\mathrm{av}}$ \\
\hline $\operatorname{CASSCF}(6,6)$ & - & - & - & -40.64 & -53.10 & -44.79 \\
\hline Strictly atomic & -41.16 & -54.19 & -45.50 & -41.30 & -54.26 & -45.62 \\
\hline \multirow[t]{3}{*}{ Delocal } & -40.57 & -53.00 & -44.71 & -40.63 & -52.83 & -44.70 \\
\hline & \multicolumn{3}{|c|}{ 1,3-Cyclobutadiene (2(A)) (2(B)) } & \multicolumn{3}{|c|}{ Cyclobutadiene $(\mathbf{2}(\mathbf{A}+\mathbf{B}))$} \\
\hline & $0.5\left(\chi_{x x}+\chi_{y y}\right)$ & $\chi_{z z}$ & $\chi_{\mathrm{av}}$ & $0.5\left(\chi_{x x}+\chi_{y y}\right)$ & $\chi_{z z}$ & $\chi_{\mathrm{av}}$ \\
\hline $\operatorname{CASSCF}(4,4)$ & - & - & - & -17.91 & -20.44 & -18.75 \\
\hline Strictly atomic & $-18.10(-17.91)$ & $-24.84(-24.87)$ & $-20.35(-20.28)$ & -18.12 & -24.84 & -20.36 \\
\hline Delocal & $-17.91(-17.72)$ & $-20.49(-17.95)$ & $-18.77(-17.80)$ & -17.92 & -20.43 & -18.76 \\
\hline
\end{tabular}


The second structure is negligible. Remarkably, the molecular properties of the resonance structures $\mathbf{2 A}$ and 2B do not differ significantly, despite differences in bonding and energy. Also, the CI response due to mixing of the two Kekule structures does not contribute to the properties.

All VB magnetisability tensors (Table 3 ) are in agreement with those evaluated at the CASSCF level. Similar to the polarisability, the magnetisability tensors for the one-structure descriptions of benzene $(\mathbf{1}(\mathbf{A}))$ and cyclobutadiene $(\mathbf{2}(\mathbf{A}) / \mathbf{2}(\mathbf{B}))$ are nearly identical to those obtained for the two-structure calculations. Small deviations, due to variation in orbitals is observed, but resonance between the two structures does not affect the magnetisability.

Regarding the parallel performance of the implementation, an excellent scaling behaviour is found for up to 16 processors. Deterioration of the performance is observed when more than 16 processors are used. This might be caused by the fact that the presented problem is fairly small. Up to 64 processors, a reasonable speedup of 42 is achieved.

\section{Conclusion}

The (parallel) implementation of a coupled valence bond theory created the opportunity to study the electric and magnetic response properties of individual Lewis structures, and the effects of resonance between them. For both benzene and cyclobutadiene, the polarisabilities and magnetisabilities are of comparable magnitude compared to those evaluated at the $\pi$-CASSCF level.

Strikingly, both the polarisability and magnetisability tensors of benzene are similar to those of its 'localised' counterpart. The CI response in this case vanishes.

\section{Acknowledgements}

Financial support from the Netherlands Organisation for Scientific Research (NWO), Grant 700.53.401, partial financial support from NWO/NCF for use of supercomputer time on TERAS/ASTER, SARA (The Netherlands, Project No. SG-032), and fruitful discussions with Dr. J.H. van Lenthe (Utrecht University) are gratefully acknowledged. Dr. D.L. Cooper (University of Liverpool) is acknowledged for providing a copy of Dr. Hyams' PhD thesis.

\section{Appendix A. Complex derivatives required for the evaluation of the magnetisability}

For magnetic field perturbations, $\hat{V}_{1}$ contains the angular momentum operator $\left(\hat{L}_{x}, \hat{L}_{y}\right.$, and $\left.\hat{L}_{z}\right)$. Note that
$\hat{V}_{1}$ is purely imaginary. Then, the first derivative with respect to complex $X$ (either an orbital rotation or a CI coefficient) becomes

$$
\left(\frac{\partial}{\partial X}\right)\left(\frac{\left\langle\Psi_{0}\left|\hat{V}_{1}\right| \Psi_{0}\right\rangle}{\left\langle\Psi_{0} \mid \Psi_{0}\right\rangle}\right)=-2\left\langle\frac{\partial \Psi_{0}}{\partial X}\left|\hat{V}_{1}-V_{1}^{0}\right| \Psi_{0}\right\rangle
$$

and the electronic Hessian (Eq. (10))

$$
\begin{aligned}
\frac{\partial^{2} E}{\partial X_{1} \partial X_{2}}= & -2\left\langle\frac{\partial^{2} \Psi_{0}}{\partial X_{1} \partial X_{2}}\left|\hat{H}-E_{0}\right| \Psi_{0}\right\rangle \\
& +2\left\langle\frac{\partial \Psi_{0}}{\partial X_{1}}\left|\hat{H}-E_{0}\right| \frac{\partial \Psi_{0}}{\partial X_{2}}\right\rangle .
\end{aligned}
$$

For the evaluation of the imaginary property gradient, the present routines for the calculation of matrix elements were adapted, so that they account for the antisymmetric nature of the property integrals.

The diamagnetic part, $\hat{V}_{2}$ contains $\left(r^{2} \mathbf{I}-\mathbf{r r}\right)[1]$. In more detail

$$
\hat{V}_{2} \subset\left(r^{2} \mathbf{I}-\mathbf{r r}\right)=\left(\begin{array}{ccc}
y^{2}+z^{2} & -x y & -x z \\
-y x & x^{2}+z^{2} & -y z \\
-z x & -z y & x^{2}+y^{2}
\end{array}\right) .
$$

\section{References}

[1] P.A. Hyams, J. Gerratt, D.L. Cooper, M. Raimondi, J. Chem. Phys. 100 (1994) 4408.

[2] J. Verbeek et al., Turtle, an Ab Initio VB/VBSCF Program, 1988-2004.

[3] J.H. van Lenthe, F. Dijkstra, R.W.A. Havenith, in: D.L. Cooper (Ed.), Valence Bond Theory, Elsevier, Amsterdam, 2002, p. 79.

[4] P.C. Hiberty, J.P. Flament, E. Noizet, Chem. Phys. Lett. 189 (1992) 259.

[5] F. Jensen, Introduction to Computational Chemistry, Wiley, Chichester, 1999.

[6] B. Levy, G. Berthier, Int. J. Quantum Chem. 2 (1968) 307.

[7] J.H. van Lenthe, G.G. Balint-Kurti, Chem. Phys. Lett. 76 (1980) 138.

[8] J.H. van Lenthe, G.G. Balint-Kurti, J. Chem. Phys. 78 (1983) 5699.

[9] J. Gerratt, I.M. Mills, J. Chem. Phys. 49 (1968) 1719.

[10] T.C. Chang, W.H.E. Schwarz, Theor. Chim. Acta (Berl.) 44 (1977) 45 .

[11] J. Almlöf, P.R. Taylor, Int. J. Quantum Chem. 27 (1985) 743.

[12] J. Verbeek, J.H. van Lenthe, Int. J. Quantum Chem. 15 (1991) 201.

[13] P.O. Löwdin, Phys. Rev. 97 (1955) 1474.

[14] F. Dijkstra, J.H. van Lenthe, J. Comput. Chem. 22 (2001) 665.

[15] M.F. Guest, I.J. Bush, H.J.J. van Dam, P. Sherwood, J.M.H. Thomas, J.H. van Lenthe, R.W.A. Havenith, J. Kendrick, Mol. Phys. 103 (2005) 719.

[16] D.L. Cooper, J. Gerratt, M. Raimondi, Nature 323 (1986) 699.

[17] Dalton, A Molecular Electronic Structure Program, Release 2.0, 2005. Available from: <http://www.kjemi.uio.no/software/dalton/ dalton.html>

[18] J.H. van Lenthe, R.W.A. Havenith, F. Dijkstra, L.W. Jenneskens, Chem. Phys. Lett. 361 (2002) 203.

[19] F. Dijkstra, J.H. van Lenthe, R.W.A. Havenith, L.W. Jenneskens, Int. J. Quantum Chem. 91 (2003) 566.

[20] P.A. Hyams, PhD Thesis, University of Bristol, 1990. 